OPEN

SUBJECT AREAS:

PHYSICS

OPTICS AND PHOTONICS

Received

23 April 2014

Accepted

19 August 2014

Published

15 September 2014

Correspondence and requests for materials should be addressed to A.L. (cqtalej@nus.edu.

sg)

\title{
Near-space flight of a correlated photon system
}

\author{
Zhongkan Tang', Rakhitha Chandrasekara', Yau Yong Sean', Cliff Cheng' ', Christoph Wildfeuer² \\ \& Alexander Ling'
}

\author{
${ }^{1}$ Centre for Quantum Technologies, National University of Singapore, Block S15, 3 Science Drive 2, 117543 Singapore, \\ ${ }^{2}$ Kantonsschule Sursee, Moosgasse 11, CH-6210 Sursee, Switzerland.
}

We report the successful test flight of a device for generating and monitoring correlated photon pairs under near-space conditions up to $35.5 \mathrm{~km}$ altitude. Data from ground based qualification tests and the high altitude experiment demonstrate that the device continues to operate even under harsh environmental conditions. The design of the rugged, compact and power-efficient photon pair system is presented. This design enables autonomous photon pair systems to be deployed on low-resource platforms such as nanosatellites hosting remote nodes of a quantum key distribution network. These results pave the way for tests of entangled photon technology in low earth orbit.

orrelated photon pair sources based on spontaneous parametric down conversion (SPDC) are an estab-
lished technology ${ }^{1}$. Since pioneering demonstrations the brightness and quality of the photon pair corre-
lations have been steadily improving ${ }^{2-7}$. The most popular application of SPDC sources is the generation of photon pairs described by a quantum entangled state.

Ongoing research on SPDC sources is largely spurred by interest in quantum communication. In particular, quantum key distribution (QKD) based on entangled photon pairs continues to attract attention due to superior privacy guarantees provided by fundamental quantum mechanics ${ }^{8-11}$. Entanglement distribution can take place via optical fiber or free-space links but current fiber technology imposes a practical distance limit due to loss and decoherence. These limitations suggest that QKD networks beyond metropolitan-scale distances will rely on freespace links ${ }^{12}$. By coupling photon pair sources to optical transmitters and placing them aboard high altitude airor space-craft, it is possible to beam entangled photons to widely separated receivers. Research on planet-wide entanglement distribution using space-craft is ongoing ${ }^{13}$ and a major milestone in this direction would be the demonstration of a bright entangled photon pair source in low earth orbit (LEO).

We propose that an entangled photon source can be demonstrated cost effectively in LEO using small spacecraft called nanosatellites ${ }^{14}$. In particular the CubeSat standard ${ }^{15}$ is very attractive due to its short design cycle, the use of commercial-off-the-shelf components and a common deployment mechanism. The standard CubeSat unit is a $1 \mathrm{~kg}, 100 \mathrm{~mm}$ cube (1U), and these units can be stacked to build larger space-craft.

The disadvantage of using CubeSats is the limited availability of resources. Restrictions on the payload of a typical $1 \mathrm{U}$ CubeSat are the form factor $\left(100 \times 100 \times 30 \mathrm{~mm}^{3}\right)$, mass $(300 \mathrm{gm})$ and available power $(2 \mathrm{~W}$ continuous). Other challenges include mechanical vibration experienced during a rocket launch, lack of shielding against radiation, possible thermal fatigue due to lack of insulation material and limited power for temperature regulation.

In response to these challenges we have designed a small, rugged and power efficient system for generating and monitoring polarization correlations between photon pairs. The system is packaged into a device whose form factor and power requirements are consistent with the payload limits of a $1 \mathrm{U}$ CubeSat. Within the package are closely integrated optical and electronic sub-systems. The electronics segment has the complete infrastructure for carrying out photon pair counting experiments and is fully automated.

The source emits classically correlated photon pairs based on type I collinear SPDC in a single crystal. To validate the key parameters of ruggedness and power efficiency it is sufficient to generate and monitor strong classical correlation between pairs of photons. If the design is able to support a basic SPDC source it can be extended to generate polarization entangled photon pairs ${ }^{6}$.

The photon pair source and detectors are housed within a machined aluminium optical unit that is black anodized to minimize internal reflection. The layout is shown to scale in Fig. 1. The optical pump is a continuous wave single mode $405 \mathrm{~nm}$ laser diode (Ondax CP-405). It has an internal volume holographic grating to ensure 


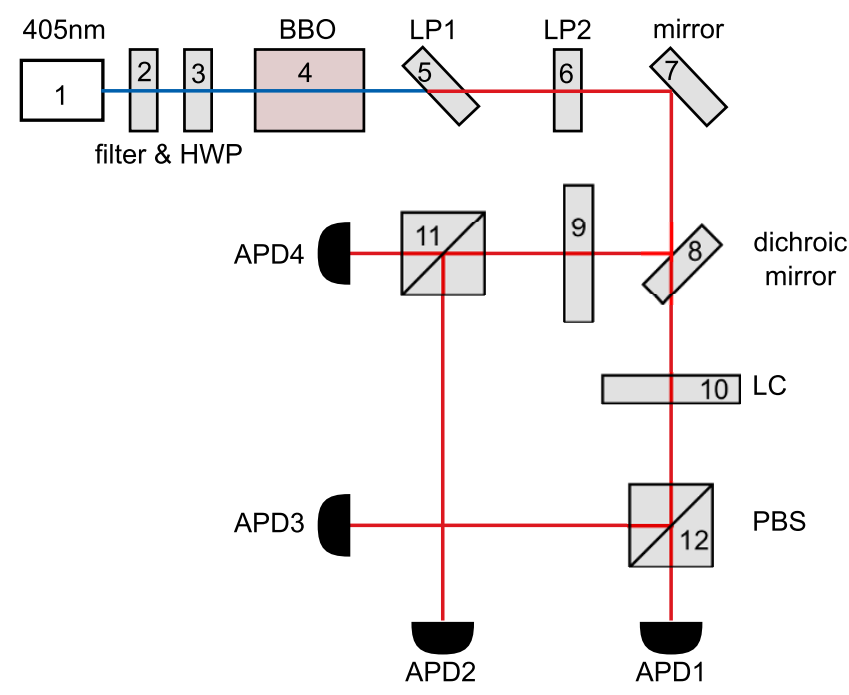

(a)

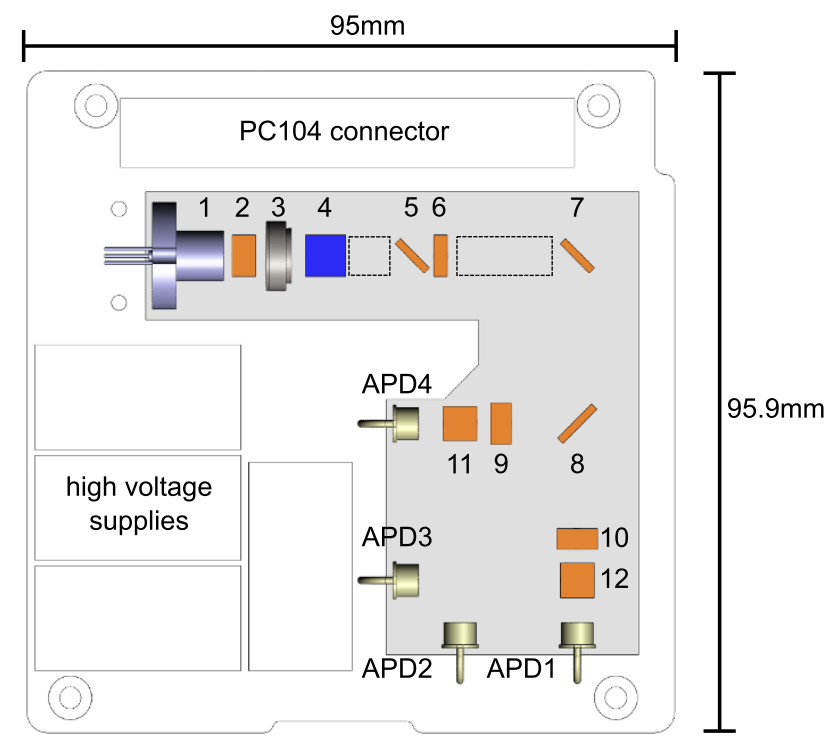

(b)

Figure $1 \mid$ Schematic of elements in the optical subsystem. Fig. 1(a) Correlated photon pairs at 760 and 867 nm are generated via type I SPDC through a $\beta$-barium-borate (BBO) crystal pumped by a $405 \mathrm{~nm}$ laser diode. The pump spectrum and polarization mode is prepared by an appropriate band-pass filter and half-wave plate (HWP). Excess pump light is removed via long pass filters (LP1 \& LP2). The liquid crystal (LC) units and polarizing beam splitters (PBS) are used for rotating and analyzing photon pairs. Two pairs of silicon avalanche photodiodes (APDs) provide redundancy in the detectors. Each APD has individual spatial and spectral filters and an individual high voltage supply. The optical unit is placed on a printed circuit board.

Fig. 1(b) is drawn to scale with $1: 1.7$ ratio, showing the position of the essential elements within the aluminium housing (grey). These are labeled by number for cross referencing. The dashed boxes are placeholders for additional crystals to enable production of polarization entangled photon pairs ${ }^{6}$. The electronic sub-system is placed on the reverse side of the board. The completed device does not exceed $30 \mathrm{~mm}$ in height.

wavelength stability over a large temperature range. Signal and idler photons are separated by a dichroic mirror and detected by silicon avalanche photodiodes (APD).

The downconversion process is carried out in a $\beta$-barium-borate (BBO) crystal $\left(5 \times 5 \times 5 \mathrm{~mm}^{3}\right)$ cut for type I phase matching at $28.78^{\circ}$. An advantage of using $\mathrm{BBO}$ in low resource systems is its relative tolerance to changes in temperature - this is useful aboard CubeSats where fine temperature control is a challenge. The designed angle cut enables collinear pair production of horizontally polarized photon pairs at 760 (signal) and $867 \mathrm{~nm}$ (idler). Excess pump light is removed via appropriate filters. Signal and idler photons are separated by a dichroic mirror (transmits idler) and detected by APDs (Laser Components SAP500). A mirror folds the optical path to satisfy the size constraint imposed by the nanosatellite platform.

Polarization correlation between signal and idler is measured using a combination of liquid crystal (LC) polarization rotators and polarizing beam splitter (PBS) cubes. Using LC devices minimizes power and space requirements. It also reduces undesirable torque that may destabilize the orientation of the nanosatellite. The polarization rotators are wavelength optimized for our source and have an extinction ratio larger than $100: 1$. The PBS cubes provide redundancy in the detectors by allowing the use of two pairs of APDs. In any single experiment, a fixed pair of APDs is used (1\&4 or $2 \& 3$ ) to conserve electrical power (power budget is available in the Supplementary Materials).

A tool-kit for precise alignment of crystals within the optical unit was developed. Once the crystals are aligned they are secured to the optical unit by epoxy. The optical unit is made light-tight by opaque polyacetal (Delrin) walls containing all laser emission. The complete optical unit (Fig. 1(b)) weighs less than 120 gm. The optical design has sufficient space for placement of additional crystals to convert this source into an entangled photon pair source ${ }^{6}$.

The LC rotators are calibrated before insertion into the optical unit. When performing a single data run for APDs $1 \& 4$, the LC device for the idler photon is supplied with a voltage that enables maximum transmission of horizontally polarized photons to the idler detector (APD1). The rotator for the signal photon is then stepped through a series of voltages that correspond to a full polarization rotation of $2 \pi$. The rotator is allowed to stabilize at each new voltage step for $0.3 \mathrm{~s}$ before data is collected. When operating the APDs 2\&3 the same protocol is used except that the idler LC device is tuned to maximize transmission to APD3.

A typical cycle for APDs $1 \& 4$ is shown in Fig. 2 when the pump is operated at $9 \mathrm{~mW}$. Approximately 4500 photon pairs are detected when the signal and idler counts per second are 360,000 and 330,000 respectively. The maximum number of corrected coincidence events

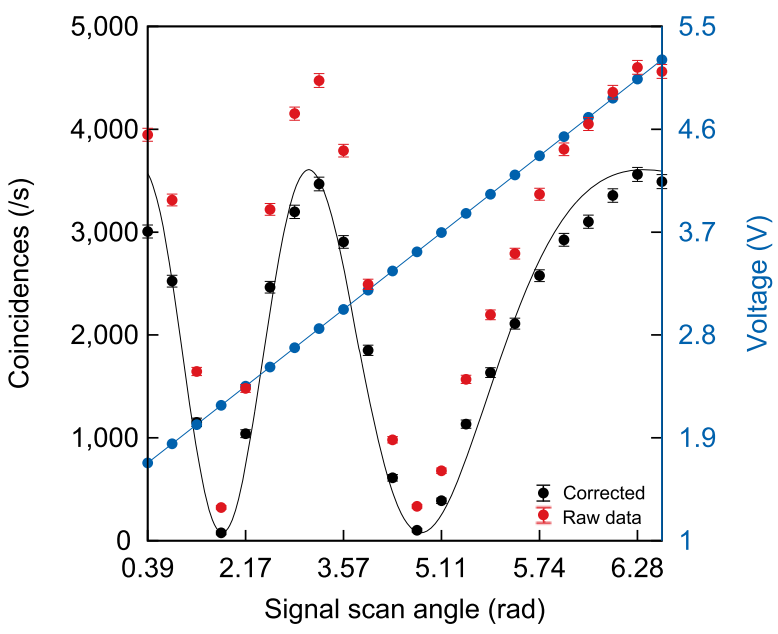

Figure $2 \mid$ Typical data set for one complete measurement where the polarization of the signal photons is rotated over $2 \pi$. The range of $2 \pi$ is achieved by increasing the voltage to the liquid crystal rotator from $1.7 \mathrm{~V}$ to $5.2 \mathrm{~V}$ (blue trace). A retarded sinusoid fitted to the corrected data has a visibility (contrast) of $95 \pm 1 \%$. 


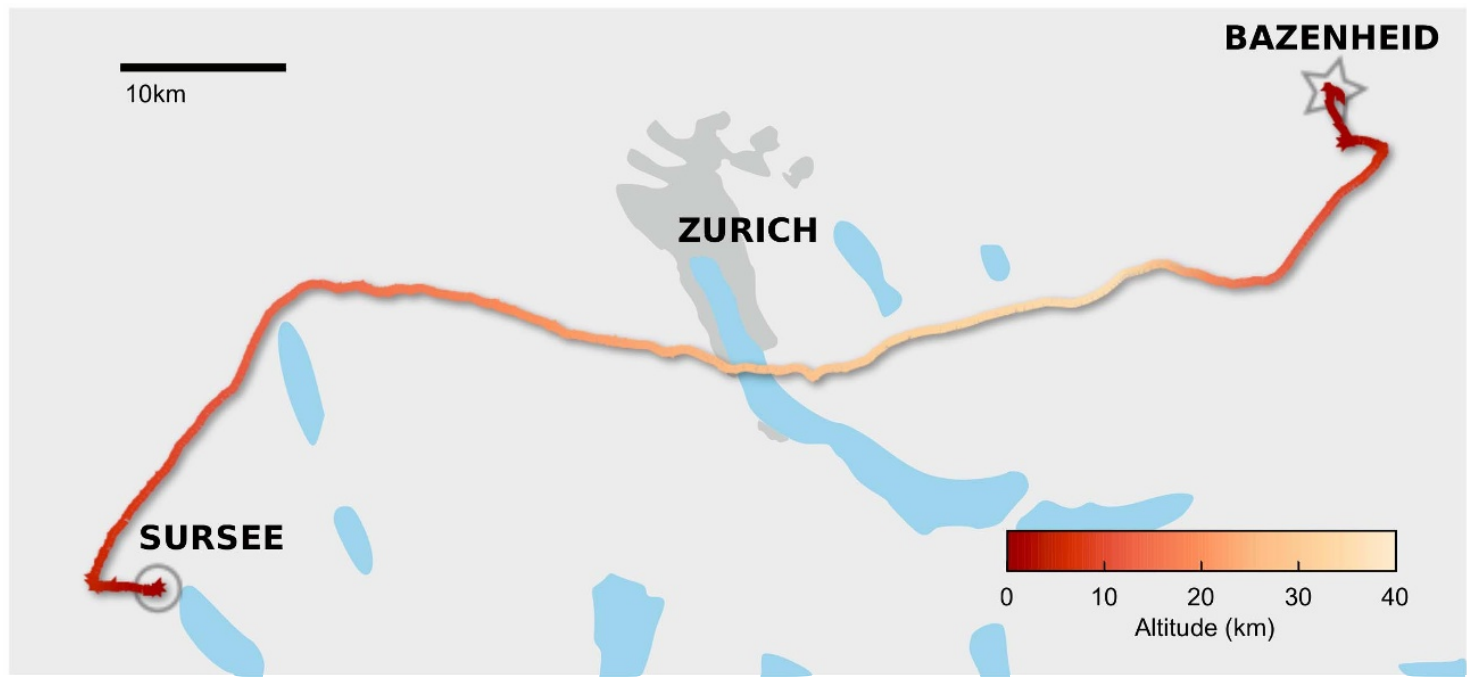

Figure 3 Flight path of the helium balloon hosting the device under test. The balloon reached a maximum altitude of $35.5 \mathrm{~km}$ before bursting. Software used: Gnuplot. Map data: Natural Earth.

is about 3600 per second. The correction is performed using the standard technique of subtracting the estimated accidental coincidences from the data. The accidental coincidences are determined at each data point by taking the product of the single photon rates and the coincidence time window. The timing window used in our setup is $9 \mathrm{~ns}$ (a limitation imposed by the low-power electronic components).

A sinusoid can be fitted to the corrected correlation data. The visibility (contrast) obtained from the sinusoid indicates the quality of the polarization correlations. In Fig. 2, the visibility from the corrected data is $95 \pm 1 \%$. This visibility value is consistent with values obtained in the alignment stage when the photons are coupled into single-mode fibers (with a collection efficiency of $20 \%$ ). Thus, we use the corrected visibility to monitor the long-term performance of the compact source. The time taken to complete the entire data collection is less than $30 \mathrm{~s}$. This design enables photon pair sources to undergo fast and low power self-certification of correlation quality.

The existing design has a pair-to-singles ratio of approximately $1 \%$. The largest contribution to this value is the poor overlap (39\%) of the detector active area (dia. $0.5 \mathrm{~mm}$ ) with the collimated pump mode (dia. $0.8 \mathrm{~mm}$ ). Detector inefficiency and imperfect transmission of devices (e.g. LC rotators) account for most of the remaining loss. Overlap between detector and pump mode can be greatly increased with the use of collection optics but is not implemented in the current design due to form factor limits. CubeSats sizes larger than $1 \mathrm{U}$ can relax the restriction but for increased launch opportunities we have designed for the smallest nanosatellite.

The electronic sub-system is built around a Programmable System-on-Chip (Cypress PSoC3). The PSoC3 provides a microcontroller integrated with a large number of mixed signal processing functions. This reduces design complexity and power consumption while maintaining functionality. The embedded micro-controller enables rapid development of the automation software needed to operate the photon pair source and data collection. The integrated electronics and optical system has a total mass below $250 \mathrm{gm}$ and requires less than $1.5 \mathrm{~W}$ to operate. A detailed description of the electronics sub-system and the results of space qualification tests are available in the supplementary material.

To investigate the performance of the system close to the target operating environment (400 km LEO) a helium filled weather balloon was used to lift the device into near-space. In addition to the device, the balloon carried tracking and telemetry instrumentation such as a GPS receiver that broadcast its position on an amateur radio band (144.8 MHz) at all times. Sensors recording 3-axis acceleration,

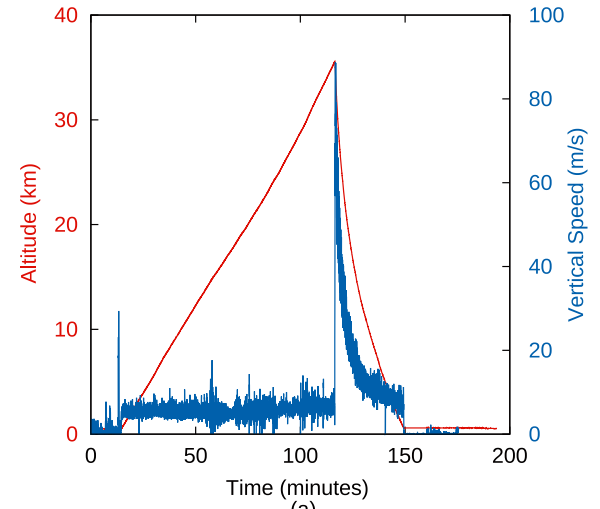

(a)

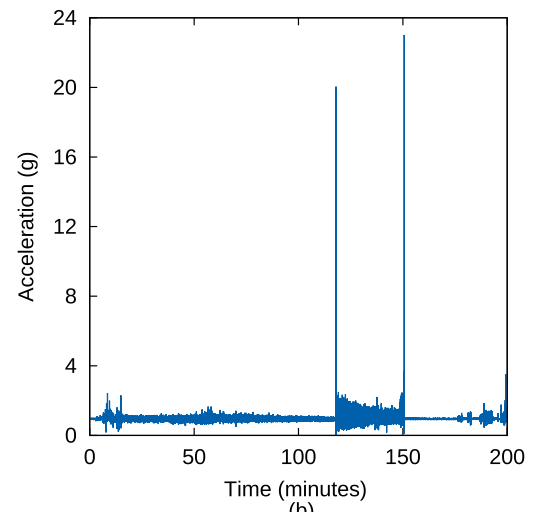

(b)

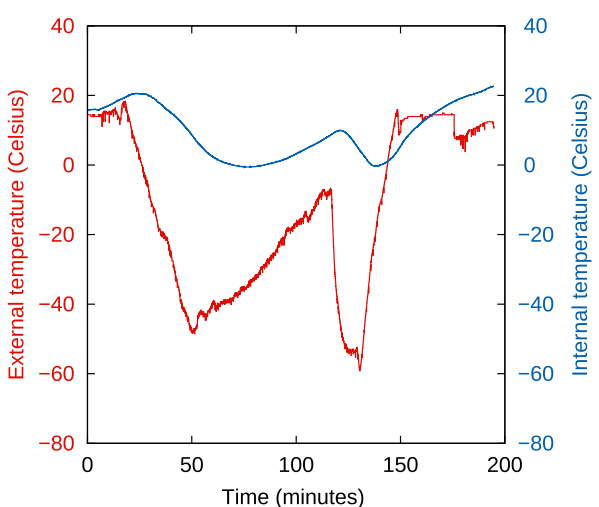

(c)

Figure 4 Conditions experienced by the photon pair system during the test flight. (a) The system was tested up to $35.5 \mathrm{~km}$ which is $10 \%$ of the planned space mission altitude. (b) The raw data from the accelerometer in the balloon package. There is a constant background of $1 \mathrm{~g}$ due to gravity. Acceleration events over $20 \mathrm{~g}$ were experienced when the helium balloon burst and the package landed. Increased acceleration due to tumbling is observed during the descent. (c) The troughs of the temperature profiles correspond to the balloon ascending and descending through the jet stream where it reached horizontal speeds up to $32 \mathrm{~ms}^{-1}$. 


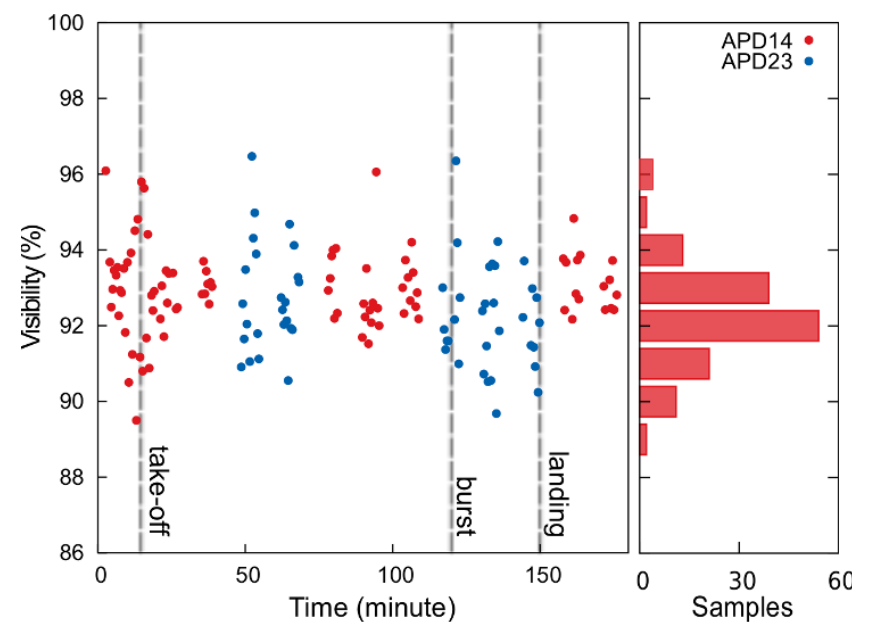

Figure $5 \mid$ Polarization correlations during test flight. The average visibility of the correlations is $92 \pm 1 \%$ (within the error of laboratory demonstrations).

air temperature, relative humidity and pressure were packaged with the correlated system, controlled by a separate micro-controller. The overall mass of the balloon package was around $2 \mathrm{~kg}$.

The device was activated on the ground 15 minutes before the balloon was released. The release point was in Sursee, Switzerland ( $\mathrm{N} 47.17^{\circ}, \mathrm{E} 8.1^{\circ}$ ) and the device landed in Bazenheid, Canton St. Gallen $130 \mathrm{~km}$ away $\left(\mathrm{N} 47.42^{\circ}, \mathrm{E} 9.1^{\circ}\right)$. The flight path is plotted in Fig. 3 and environmental data from the flight are plotted in Fig. 4. The test flight took around 2 hours and reached a ceiling altitude of $35.5 \mathrm{~km}$ above sea level before bursting. The rate of ascent was approximately $5 \mathrm{~ms}^{-1}$ and after the balloon burst the maximum descent velocity was over $90 \mathrm{~ms}^{-1}$. When the air density is sufficiently high, an attached parachute slowed the descent to below $10 \mathrm{~ms}^{-1}$.

Total system acceleration experienced by the device is plotted in Fig. 4(b). The accelerometer (Analog Devices ADXL345) measures up to $16 \mathrm{~g}$ per axis with 13-bit resolution. The early acceleration events are due to the initial drag from the weather balloon during take off. Two major acceleration events occur after 120 and 150 minutes corresponding to the balloon burst (20 g) and landing $(23 \mathrm{~g})$ respectively, in an otherwise uniform plot.

The internal and external temperature profiles are correlated (Fig. 4(c)). The troughs of the temperature profiles corresponds to the balloon ascending and descending through the jet stream. The internal temperature varies from $0^{\circ} \mathrm{C}$ and $20^{\circ} \mathrm{C}$ in a low pressure environment (below 3 mbar at $35.5 \mathrm{~km}$ altitude).

During flight the APD pairs were operated alternately so that the performance for all detectors could be monitored. The average visibility throughout the experiment is around 92\% (Fig. 5). This nearspace test flight demonstrates that the system continues to generate and monitor polarization correlations despite significant variation in acceleration, pressure and temperature.

A compact, robust and power-efficient system for generating and monitoring polarization correlated photon pairs has been designed and assembled. A single device was subjected to qualification tests that examined the system tolerance to vacuum, temperature and vibration. It was then placed on a weather balloon and lifted $35.5 \mathrm{~km}$ into the upper atmosphere to test its performance under near-space conditions.

The test results demonstrate that the integrated source and detector package survives conditions that most laboratory-based setups cannot tolerate. The ultimate aim is to operate the device at an LEO altitude of about $400 \mathrm{~km}$. The balloon test has enabled the device to be tested to almost $10 \%$ of that altitude. The combined suite of tests demonstrate that the system is at a high level of technological readiness paving the way for space-based demonstration of entangled photon sources.

In future work additional crystals will be integrated so that the source generates polarization entangled photon pairs. Different SPDC materials may be integrated into the device with minimal changes to the form factor and power requirement.

In overcoming the resource limitations encountered on nanosatellites our design enables photon pair sources to be deployed over a larger number of low-resource operating environments. It is anticipated that they will be deployed not only on satellites, but also on small unmanned aerial vehicles or even for handheld communicators. The integration of measurement apparatus for monitoring the polarization correlations also serve as a self-diagnostic tool for analyzing the status of the source. Rugged and efficient photon pair sources capable of self-diagnosis are highly desirable as nodes in any quantum network whether in space or on Earth.

1. Burnham, D. C. \& Weinberg, D. L. Observation of simultaneity in parametric production of optical photon pairs. Phys. Rev. Lett. 25, 84-87 (1970).

2. Ou, Z. Y. \& Mandel, L. Violation of Bell's inequality and classical probability in a two-photon correlation experiment. Phys. Rev. Lett. 61, 50-53 (1988).

3. Kwiat, P. G. et al. New high-intensity source of polarization-entangled photon pairs. Phys. Rev. Lett. 75, 4337-4341 (1995).

4. Kwiat, P. G., Waks, E., White, A. G., Appelbaum, I. \& Eberhard, P. H. Ultrabright source of polarization-entangled photons. Phys. Rev. A 60, R773-776 (1999).

5. Kurtsiefer, C., Oberparleiter, M. \& Weinfurter, H. High-efficiency entangled photon pair collection in type-II parametric fluorescence. Phys. Rev. A 64, 023802/1-4 (2001).

6. Trojek, P. \& Weinfurter, H. Collinear source of polarization-entangled photon pairs at nondegenerate wavelengths. Appl. Phys. Lett. 92, 211103/1-3 (2008)

7. Steinlechner, F. et al. A high-brightness source of polarization-entangled photons optimized for applications in free space. Opt. Express 20, 9640-9649 (2012).

8. Ekert, A. K. Quantum cryptography based on Bell's theorem. Phys. Rev. Lett. 67, 661-663 (1991).

9. Gisin, N., Ribordy, G., Tittel, W. \& Zbinden, H. Quantum cryptography. Rev. Mod. Phys. 74, 145-195 (2002).

10. Gisin, N. \& Thew, R. Quantum communication. Nature Photon. 1, 165-171 (2007).

11. Acin, A. et al. Device-independent security of quantum cryptography against collective attacks. Phys. Rev. Lett. 98, 230501/1-4 (2007).

12. Ursin, R. et al. Entanglement-based quantum communication over $144 \mathrm{~km}$. Nature Phys. 3, 481-486 (2007).

13. Jennewein, T. \& Higgins, B. The quantum space race. Phys. World 26, 52-56 (2013).

14. Morong, W., Oi, D. \& Ling, A. Quantum optics for space platforms. Opt. and Photon. News 23, 42-49 (2012).

15. Woellert, K., Ehrenfreund, P., Ricco, A. J. \& Hertzfeld, H. Cubesats: cost-effecitve science and technology platforms for emerging and developing nations. $A d v$. Space Res. 47, 663-684 (2011).

\section{Acknowledgments}

We thank Tan Yue Chuan for his assistance in preparing the test devices and James Grieve for manuscript assistance. Invaluable assistance was provided by Jens Spinner and the Radio Club Sursee in tracking and recovering the balloon test package. The CQT team also thanks Daniel Oi for technical discussions regarding CubeSat technology. C. Cheng is supported by a DSO-CQT(NUS) grant.

\section{Author contributions}

A.L. conceived the concept of the test flight. Y.Y.S. and A.L. co-designed the alighment tool-kit and mechanical structures. C.C. and A.L. co-designed the electronics control system. T.Z. and A.L. prepared the optical source. R.C. prepared the environmental sensors. Y.Y.S. and R.C. conducted the space qualification tests. C.W. prepared the flight logistics and materials. C.C., T.Z. and C.W. conducted the flight test. The text was written collaboratively by all the authors.

\section{Additional information}

Supplementary information accompanies this paper at http://www.nature.com/ scientificreports

Competing financial interests: The authors declare no competing financial interests.

How to cite this article: Tang, Z. et al. Near-space flight of a correlated photon system. Sci. Rep. 4, 6366; DOI:10.1038/srep06366 (2014). 
cc)(i) (2) This work is licensed under a Creative Commons Attribution-NonCommercialShareAlike 4.0 International License. The images or other third party material in this article are included in the article's Creative Commons license, unless indicated otherwise in the credit line; if the material is not included under the Creative
Commons license, users will need to obtain permission from the license holder in order to reproduce the material. To view a copy of this license, visit http:// creativecommons.org/licenses/by-nc-sa/4.0/ 